\title{
Improving circulatory death donor heart function: A novel approach
}

Mohammed Quader, MD, ${ }^{\mathrm{a}, \mathrm{b}}$ Eleonora Mezzaroma, PhD, ${ }^{\mathrm{a}, \mathrm{c}, \mathrm{d}}$ Niluka Wickramaratne, MD, ${ }^{\mathrm{b}}$ and Stefano Toldo, $\mathrm{PhD}$, , , ,d Richmond, Va

From the ${ }^{\mathrm{a}}$ Pauley Heart Center, ${ }^{\mathrm{b}}$ Department of Surgery, ${ }^{\mathrm{c}}$ Johnson Center for Critical Care and Pulmonary Research, and ${ }^{\mathrm{d}}$ School of Pharmacy, Virginia Commonwealth University, Richmond, Va.

Funded by American Heart Association Award No. 16SDG31080002, a Veterans Administration Merit Review (Grant ID: CARA-015-17S, Award No. I01 BX003859), and a Pauley Heart Center Pilot Award to Dr Quader, and Virginia Commonwealth University's Clinical and Translational Science Awards (UL1TR000058 from the National Center for Advancing Translational Sciences-Center for Clinical and Translational Research Endowment Fund) to Dr Toldo.

Disclosures: The authors reported no conflicts of interest.

The Journal policy requires editors and reviewers to disclose conflicts of interest and to decline handling or reviewing manuscripts for which they may have a conflict of interest. The editors and reviewers of this article have no conflicts of interest.

Received for publication Feb 8, 2021; accepted for publication June 7, 2021; available ahead of print June 12, 2021.

Address for reprints: Mohammed Quader, MD, Department of Surgery, Virginia Commonwealth University, 1200

E Broad St, Richmond, VA 23298 (E-mail: mohammed.quader@vcuhealth.org).

JTCVS Techniques 2021;9:89-92

2666-2507

Copyright (c) 2021 The Author(s). Published by Elsevier Inc. on behalf of The American Association for Thoracic Surgery. This is an open access article under the CC BY-NC-ND license (http://creativecommons.org/licenses/bync-nd/4.0/).

https://doi.org/10.1016/j.xjtc.2021.06.018

Heart transplantation (HTx) is limited due to the availability of donor hearts. ${ }^{1}$ Up to $20 \%$ of patients die awaiting HTx. ${ }^{1}$ HTx numbers could increase by $56 \%$ if hearts donated after circulatory death (DCD) were used for transplantation. ${ }^{2}$ Recent trials have shown that DCD heart transplantation is feasible. ${ }^{3}$ However, innate to the DCD process is ischemia, ventricular wall stretching, and high catecholamine release. Upon implantation, reperfusion injury causes additional damage to the DCD heart.

Myocardial ischemia/reperfusion injury activates the NACHT, LRR, and PYD domains-containing protein-3 (NLRP3) inflammasome, an established mediator of ischemic injury and pathological remodeling. ${ }^{4,5}$ We studied the cardioprotective effects of NLRP3 inhibition in a DCD rat heterotopic HTx model.

\section{METHODS}

Our protocol (Figure 1) followed the Guide for the Care and Use of Laboratory Animals (National Institutes of Health Publication No. 86-23) and was approved by the Virginia Commonwealth University Institutional Animal Care and Utilization Committee.

Sprague-Dawley rats (aged 8-10 weeks) were anesthetized (ketaminexylazine, $100-10 \mathrm{mg} / \mathrm{kg}$ ), intubated, connected to a ventilator, and anticoagulated with heparin $(1000 \mathrm{U} / \mathrm{kg})$. A catheter was placed in the right carotid artery; vecuronium-bromide $(1.2 \mathrm{mg} / \mathrm{kg})$ was administered to paralyze the diaphragm. After 1 minute, the ventilator was disconnected to initiate DCD process. After 10 minutes, the heart was exposed, and the aorta was clamped distal to the brachiocephalic branch. Ice-cold University of Wisconsin (UW) solution (12 mL) was perfused through the carotid catheter to flush and cool the heart. To the treatment group of DCD hearts, NLRP3 inhibitor (NLRP3-I) 16773-34-0 (Sigma-Aldrich, St Louis, Mo) $(50 \mu \mathrm{M})$ was added to the UW solution. Warm ischemia time (ie, time

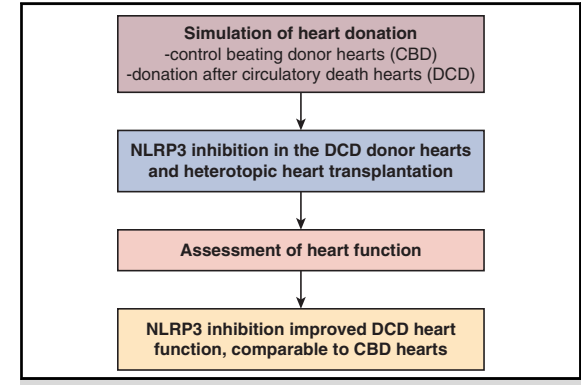

Schematic representation of the heterotopic transplantation and functional assessment of transplanted hearts. Rats were randomized to CBD or DCD donor groups. The anesthetized donor rats were intubated, ventilated, and the left carotid artery was cannulated to deliver a paralytic agent. Following the CBD or DCD protocol, the hearts were procured and preserved with the UW solution alone or UW solution with $\mathrm{NLRP}_{3}$ inhibitor added to it. The arrow represents the donor heart being placed into the recipient's abdomen. The procured heart was transplanted into the recipient rat's abdomen, which was also pretreated with NLRP3-I or not. The dotted lines with arrows represent 3 groups of transplanted hearts, placed against each panel of measured develop pressure. The graft function measured after 24 hours of transplantation is represented against each group- $C B D$, $D C D$, and DCD + NLRP $3-I$. Developed pressure is measured on the $y$-axis scaled to $200 \mathrm{~mm} \mathrm{Hg}$. The upper third of the panel depicts a normal developed pressure from the control group of hearts. The middle third of the panel represents the decreased developed pressure in untreated DCD hearts. The lower third of the panel represents DCD hearts treated with $\mathrm{NLRP}_{3}$ inhibitor. They produced a strong developed pressure that matched the control group of hearts.

\section{CENTRAL MESSAGE}

Heart transplantation numbers

can be increased by utilizing

donation after circulatory death

hearts treated with NLRP3

inhibitor.

See Commentaries on pages 93 and 95

between ventilatory support withdrawal and UW solution infusion) for DCD hearts was 25 minutes. In the control beating-heart donor (CBD) group, ventilation was continued until cardiac arrest with UW solution without the ischemia period. Following infusion of UW solution, solution 

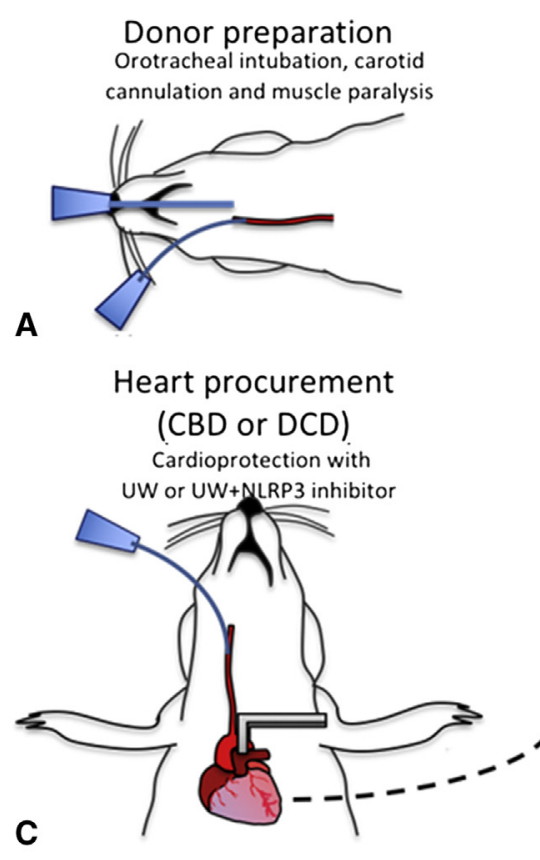

\section{Reanimation of the} transplanted heart

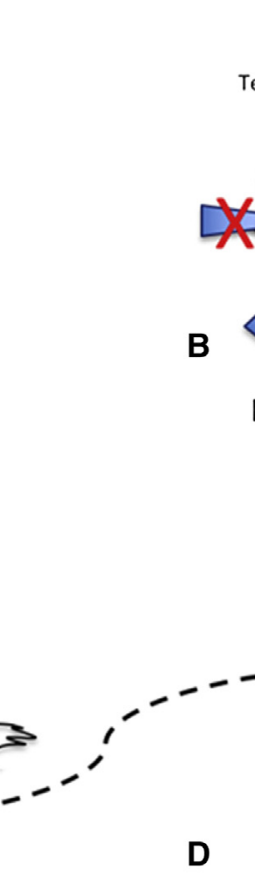

.
DCD initiation

Termination of ventilatory support

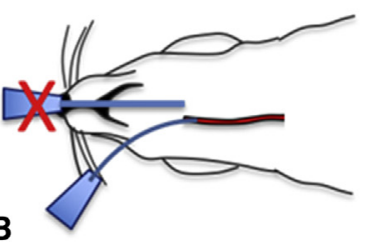

Heterotopic DCD heart

transplantation

Recipient pre-treated with or without the NLRP3 inhibitor

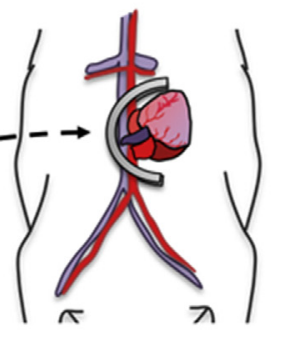

Assessment of graft function after 24 hours

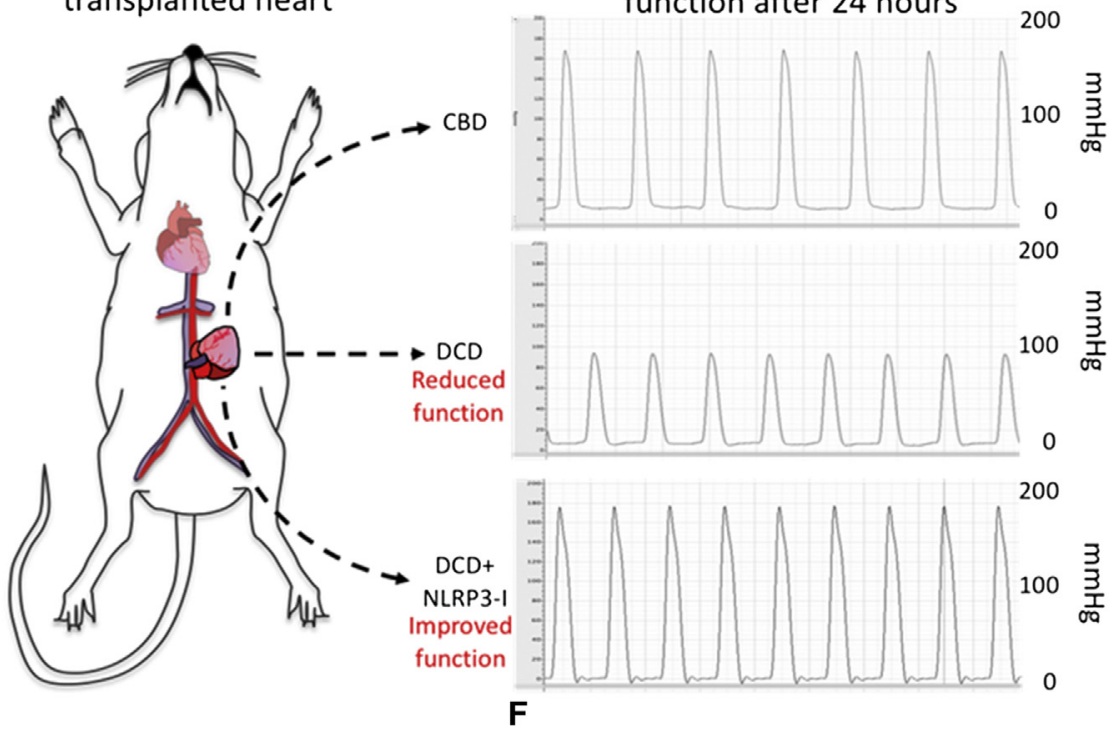

FIGURE 1. Major steps of the heterotopic transplantation and functional assessment of transplanted hearts. A, The anesthetized donor rat is intubated, ventilated, and the left carotid artery is cannulated to deliver a paralytic agent. B, To induce the donation after circulatory death (DCD) process, the ventilatory support is terminated. Shown as an $X$ mark on the endotracheal tube. C, At the time of procurement, the ascending aorta was clamped distal to the innominate artery, shown as a grey right-angle clamp, and ice-cold University of Wisconsin (UW) solution was delivered through the left carotid artery into the coronary circulation. In a group of DCD hearts, the UW solution was supplemented with the NACHT, LRR, and PYD domains-containing protein-3 (NLRP3) inhibitor (NLRP3-I). Control beating-heart donor (CBD) hearts were procured without ischemia. D, The procured heart was transplanted into the recipient rat's abdomen, which were also pretreated with NLRP3-I or not. A U-shaped vascular clamp, shown as a grey half-circle overlying abdominal aorta and inferior vena cava, was used to isolate a segment of the recipient's abdominal aorta and vena cava. The vascular anastomosis was performed between donor aorta to recipient aorta and donor pulmonary artery to the recipient vena cava. The dotted arrow line between panels $\mathrm{C}$ and $\mathrm{D}$ represents the donor heart being transplanted into the abdomen of the recipient rat. E, Upon removing the vascular clamp and reestablishment of coronary circulating, the engrafted heart resumed contractions. Following hemostasis, the abdomen was closed in 2 layers of nonabsorbable suture and rats were recovered. After 24 hours, rats were anesthetized and transplanted hearts exposed by opening the abdominal wall. The transplanted heart function was measured with a balloon-tipped catheter introduced in the left ventricle transduced to software, allowing the continuous recording of developed pressure, $\pm \mathrm{dP} / \mathrm{dt}$. The 3 dotted lines with an arrow represent three study groups of transplanted hearts. F, The graft function measured after 24 hours of transplantation is represented against each group: CBD, DCD, and DCD + NLRP3-I. Developed pressure is measured on the $y$-axis scaled to $200 \mathrm{~mm} \mathrm{Hg}$. The upper third of the panel depicts a normal robust developed pressure from the control group of hearts. The middle third of the panel represents the decreased developed pressure in untreated DCD hearts. The lower third of the panel represents DCD hearts treated with NLRP3-I. They produced a strong developed pressure that matched the control group of hearts. 


\section{Developed pressure}

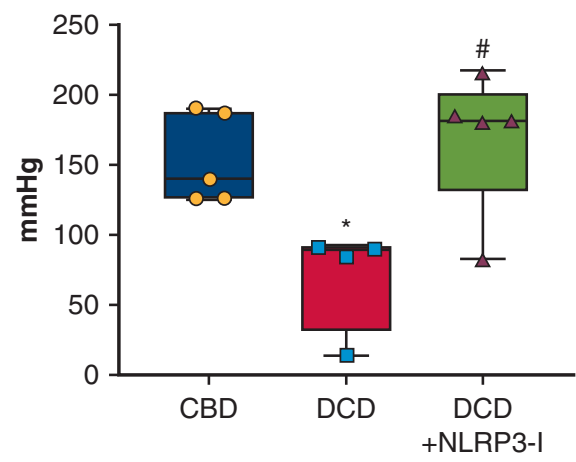

A
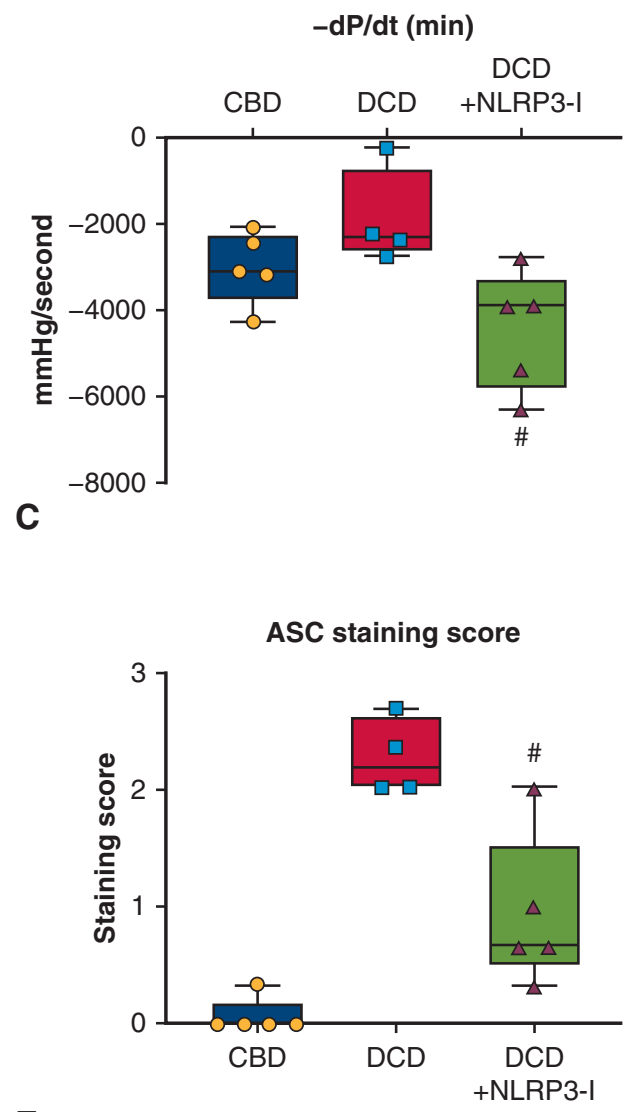

E

FIGURE 2. Cardiac function of engrafted control donor hearts and donation after circulatory death hearts with or without inhibition of NACHT, LRR, and PYD domains-containing protein-3 (NLRP3). A through D, Developed pressure, $+\mathrm{dP} / \mathrm{dt}(\mathrm{max})$, and $-\mathrm{dP} / \mathrm{dt}(\mathrm{min})$ and rate pressure product that were assessed 24 hours after heterotopic heart transplantation of control beating-heart donor (CBD) hearts or donation after circulatory death (DCD) hearts, with or without the use of the NLRP3 inhibitor (NLRP3-I). The measurements are expressed as mean and standard error of the mean. E and F, The expression of apoptosisassociated speck-like protein containing a caspase-recruiting domain (ASC), a marker of inflammasome activation in the explanted heart tissue using a double immunofluorescence technique. Cardiomyocytes were stained with a second primary antibody against cardiac alpha-actin (1:200; Sigma-Aldrich) revealed by a secondary antibody anti-mouse Alexa Fluor 488-conjugated (1:100; Invitrogen, Waltham, Mass). Nuclei counterstaining was performed with 4',6- diamidino-2-phenylindole (DAPI) 1: 20,000 for 5 minutes. A negative control with nonspecific immunoglobulin G was used as reference. The resulting images (400× magnification) were acquired through Olympus IX70 microscope, and analyzed with CellSens software (Olympus life Science, Waltham, Mass). Green stain represents actin filaments; nucleus is stained in blue color and ASC protein stained as reddish purple. The ASC quantification was performed using a semiquantitative scale ranging from 0 (no expression) to $1+$ (low expression, $<1$ per high power field), 2+ (moderate, 1-9 aggregates per high power field or diffuse stain with few aggregates) or $3+$ (diffuse, $>10$ aggregates per high power field). ASC protein levels were not detected in CBD control hearts, significantly increased in DCD hearts and detectable but significantly less in DCD NLRP3-I group. Panes F represents no detectable ASC protein in CBD heart samples, easily noticeably ASC protein levels in DCD hearts and faintly noticeable in DCD NLRP3-I heart samples. * $P<.05$ versus CBD; \#P<.05 versus DCD. No differences were found between the CBD and DCD + NLRP3-I groups. 
hearts were procured by dividing the pulmonary artery and aorta distally, then ligating and dividing the vena cava. Hearts were then stored in icecold UW solution (5-10 minutes) for implantation.

Recipients (5-6 per group) rats were anesthetized using isoflurane. Abdominal wall was opened in midline and retracted. The abdominal aorta and the inferior vena cava were isolated, clamped with a Ushaped Cooley vascular-clamp between the renal arteries and the aortic bifurcation. The donor aorta and pulmonary artery were sutured to the recipient abdominal aorta and vena cava, respectively with 8-0 Prolene. Cooley clamp was removed, hearts reperfused, and the abdominal wall closed to recover the rat. Slow-release buprenorphine $(1 \mathrm{mg} / \mathrm{kg})$ was administered subcutaneously. The recipients in NLRP3-I group also received 16773-34-0 (100 mg/kg, via gavage), 30 minutes before surgery.

After 24 hours, the recipients were anesthetized (using isoflurane), the transplanted hearts were exposed, and a balloon-tip-catheter was inserted in the left ventricle via mitral valve. Developed pressure and $\mathrm{dP} / \mathrm{dt}$ (change in pressure over change in time) were recorded using a Powerlab-station and Labchart-7 (AD Instruments, Colorado Springs, Colo). The heart tissue was analyzed for apoptosis-associated specklike protein containing a caspase-recruiting domain (ASC), a marker of activated NLRP3 inflammasome. The statistical analysis was performed using SPSS 24.0 package for Mac (IBM-SPSS Inc, Armonk, NY). Measurements are reported as the mean and standard error of the mean. Differences between groups were assessed using analysis of variance with post-hoc Tukey test. The Mann-Whitney test was used to determine statistical difference between ASC protein levels in DCD and DCDNLRP3 I hearts.

\section{RESULTS}

All the hearts in the CBD or DCD-NLRP3-I group remained functional at 24 hours. Two DCD grafts (without NLRP3-I) were nonfunctional at 24 hours. Compared with the CBD hearts, the DCD hearts had significantly reduced developed pressure, $+\mathrm{dP} / \mathrm{dt}(\max )$, and a nonsignificant worsening of the $-\mathrm{dP} / \mathrm{dt}(\mathrm{min})$ (Figure 2). Heart function (developed pressure and $\pm \mathrm{dP} / \mathrm{dt}$ ) in DCD NLRP3-I group was preserved and was comparable to control CBD hearts. The ASC protein levels were not detectable in CBD group, significantly elevated in DCD group compared with the DCD NLRP3-I group.

\section{DISCUSSION}

Strategies to limit ischemia/reperfusion injury in DCD hearts could facilitate their routine use for HTx. NLRP3 inflammasome is a major mediator of inflammation-mediated damage to the DCD heart. ${ }^{4}$ We demonstrated in our lab that NLRP3 knockout mice were protected from ischemia/reperfusion injury associated with the DCD process. ${ }^{4}$ In the present study, we show that inhibition of NLRP3 in DCD hearts improve the function and decreased the formation of active NLRP3 inflammasome (ie, decreased ASC protein levels in DCD NLRP3-I heart tissue). NLRP3 inflammasome inhibitor has been shown to be cardioprotective following acute myocardial infarction in mice. ${ }^{5}$ Our data confirm this observation in global ischemic hearts and expands on the in vivo effect of NLRP3 inhibition started during procurement. We only examined the heart function 24 hours following HTx; however, NLRP3 inhibitor is known to show protective effects up to 7 days following acute myocardial infarction in mice hearts. The inflammasome expression directly correlates with the severity of cellular rejection in biopsy specimens of patients who received HTx. Consequently, NLRP3-I has the potential to reduce rejection rates and improve transplantation outcomes.

\section{CONCLUSIONS}

NLRP3 inhibition improves the function of DCD hearts and may be a promising treatment to expand the heart donor pool.

\section{References}

1. Organ Procurement and Transplantation Network (OPTN) and Scientific Registry of Transplant Recipients (SRTR). OPTN/SRTR 2017 Annual Data Report. Available at: http://optn.transplant.hrsa.gov. Accessed February 8, 2021.

2. Messer S, Page A, Rushton S, Berman M, Tsui S, Catarino P, et al. The potential of heart transplantation from donation after circulatory death donors within the United Kingdom. J Heart Lung Transplant. 2019;38:872-4.

3. Messer S, Cernic S, Page A, Messer S, Cernic S, Page A, et al. A 5-year singlecenter early experience of heart transplantation from donation after circulatorydetermined death donors. J Heart Lung Transplant. 2020;39:1463-75.

4. Quader M, Mezzaroma E, Kenning K, Toldo S. Targeting the NLRP3 inflammasome to reduce warm ischemic injury in donation after circulatory death heart. Clin Transplant. 2020;34:e14044.

5. Toldo S, Abbate A. The NLRP3 inflammasome in acute myocardial infarction. Nat Rev Cardiol. 2018;15:203-14. 